\title{
Threshold-based 3D Tumor Segmentation using Level Set (TSL)
}

\author{
Sima Taheri ${ }^{\dagger}$, Sim Heng Ong ${ }^{\dagger}$, Vincent Chong ${ }^{\ddagger}$ \\ ${ }^{\dagger}$ Department of Electrical and Computer Engineering \\ ${ }^{\ddagger}$ Department of Diagnostic Radiology \\ National University of Singapore \\ Email: $\{$ sima,eleongsh,dnrcfhv\}@nus.edu.sg
}

\begin{abstract}
Three-dimensional segmentation is reliable approach to achieve a proper estimation of tumor volume. Among all possible methods for this purpose, level set can be used as a powerful tool which implicitly extracts the tumor surface. In this paper, we introduce a threshold-based algorithm for $3 D$ tumor segmentation using level set (TSL). This algorithm uses a global threshold to form the level set speed function which is updated iteratively throughout the level set growing process. An important feature of TSL is that no explicit knowledge about the tumor and non-tumor density functions is required. The proposed method can be implemented in the automatic and semi-automatic forms depending on the complexity of the tumor shape. TSL is examined on several clinical MRIs for both visual and quantitative evaluation. Experimental results demonstrate the effectiveness of our approach.
\end{abstract}

\section{Introduction}

A proper estimation of the tumor size is useful for several applications such as evaluating the effect of therapy on tumor and changing the treatment plans. Although the largest tumor diameter is widely used as an indication of the tumor size, because of three-dimensional shape of the tumor it may not reflect a proper assessment of this tumor attribute [8]. The tumor volume, on the other hand, suggests an appropriate representation of the tumor size. One way to find an estimation of tumor volume is to use segmentation algorithms. Such schemes implicitly acquire the tumor volume through extracting the 3D tumor surface.

In order for 3D image segmentation, active surfaces are widely used implicitly in the form of level set or explicitly in the form of snake. However, level set method is preferred because of its ability to handle complex geometries and topological changes. But, there are some difficulties in using level set which make it necessary to be provided by some additional knowledge about the desired regions. For example, Ho et.al. proposed the region competition idea in the level set evolution in which the difference between T1W and CET1W magnetic resonance images (MRIs) is used to estimate the parametric density functions of tumor and non-tumor regions [2]. This idea can be extended based on non-parametric density estimation using kernel expansion or Parzen windowing [7]. The main drawback of such algorithms is their dependency on estimation of tumor and nontumor probability density functions. On one hand, the parametric estimation of the density functions may not provide necessary precision because tumors are not generally appeared with the uniform intensities. On the other hand, nonparametric density estimation methods like Parzen windowing need to have enough training samples of both regions. Moreover, the additional complexity of estimation imposed to such algorithms causes a tendency toward density independent approaches.

In this paper, we introduce a threshold-based density independent approach which benefits level set for 3D brain tumor segmentation in the MRIs. The basic idea in this algorithm is to use a global threshold to form the level set speed function. The initial threshold is calculated based on the level set initialization and then updated throughout the process of segmentation, iteratively. Upon reaching the tumor boundary, because of the contrast between tumor and non-tumor intensities, the variation of the threshold declines so that the process stops. This algorithm can be implemented in the automatic and semi-automatic forms depending on the complexity of the tumor shape.

The rest of the paper is organized as follows. Sec. 2 introduces the TSL algorithm, its iterative approach for finding and updating the threshold and its stopping criterion. Furthermore, the formulation of the level set and designing its speed function are presented in this section. In Sec. 3 a detailed discussion about threshold updating is provided in addition to a modified TSL for non-homogeneous tumors. Simulation results in Sec. 4 show the effectiveness of our algorithm. Finally, Sec. 5 concludes the whole paper. 


\section{TSL algorithm}

Let us assume that the histogram of tumor and non-tumor ${ }^{1}$ regions are slightly overlapped. Such assumption is quite frequent in MR images. Without loss of generality, in this paper we consider the cases that tumor region has mean intensity value grater than that of the background. In such a situation, a threshold can be found so that it discriminates between tumor and non-tumor voxels with high probability. Using level set, TSL specifies an algorithm to find a proper threshold as well as a method to update it on an iterative basis. The first estimation of the threshold is based on the level set initialization which is performed by user inside the tumor region. The merit of TSL algorithm is that no explicit knowledge about the density functions of tumor and non-tumor regions is required.

TSL needs an appropriate estimation of the threshold to perform segmentation properly. For such an estimation, the concepts of confidence interval and confidence level must be considered. In the following, firstly we explain these concepts and use them to estimate the threshold. After that, the level set evolution equation is represented fitting to TSL and its speed function is designed on the basis of our threshold updating approach. Level Set initialization and TSL stopping criterion are also discussed in this section.

\subsection{Confidence Interval}

The Confidence Interval (CI) can be defined for any distribution as an interval in which a certain percentage, Confidence Level (CL), of observations are located. For a symmetric distribution such as normal, the $\mathrm{CI}$ is within the $k$ standard deviations, i.e., $k \sigma$, around the population mean, $\mu$. A general relation between CI and CL can be found based on the Chebyshev's Inequality. It should be noted that the Chebyshev's inequality holds without any assumption regarding the shape of the distribution. For a random variable $\xi$ with the finite mean and variance we have,

$$
P(|\xi-\mu| \geq k \sigma) \leq \frac{1}{k^{2}} \quad k>0
$$

which shows that greater than $\left(1-\frac{1}{k^{2}}\right)$ percent of population falls within $k$ standard deviations from the population mean. For the non-symmetric distributions, the one-tailed version of Chebyshev's inequality can be used as follows [6],

$$
P(\xi-\mu \geq k \sigma) \leq \frac{1}{1+k^{2}}
$$

\footnotetext{
${ }^{1}$ The area just around the tumor boundary and inside the cube which encompasses tumor. This cube is our range of interest (ROI)
}

\subsection{Threshold updating}

According to the concept of CI, we define an expression for threshold updating as follows,

$$
T_{i+1}=\hat{\mu}_{i}-k \hat{\sigma}_{i} \quad i \geq 0
$$

where $T_{i+1}$ is the threshold estimation for the $(i+1)^{t h}$ iteration and $k$ is the factor which determines the confidence level and must be chosen properly. Later, the effect of this parameter is discussed in great detail. Mean and standard deviation denoted by $\hat{\mu}_{i}$ and $\hat{\sigma}_{i}$ respectively and are calculated as follows,

$$
\begin{gathered}
\hat{\mu}_{i}=\frac{1}{n} \sum_{j=1}^{n} x_{i j} \\
\hat{\sigma}_{i}^{2}=\frac{1}{n-1} \sum_{j=1}^{n}\left(x_{i j}-\hat{\mu}_{i}\right)^{2}
\end{gathered}
$$

where $n$ is the number of accepted tumor samples, $x_{i j}$, upto $i^{t h}$ iteration. Based on the Eq. (3), in each iteration we define the threshold as a one tailed confidence interval of the accepted samples upto that iteration.

\subsection{Level Set speed function design}

In the level set based segmentation, introduced in [1,3], a surface, $\gamma(t)$, is embedded as a zero level set of a higher dimensional function $\phi(\mathbf{x}, t)=d\left(\mathbb{R}^{3} \times \mathbb{R}^{+} \rightarrow \mathbb{R}\right)$, where $d$ is the signed distance from $\mathbf{x}$ to $\gamma(t)$. The evolution equation of the level set is defined as follows [5, 9],

$$
\frac{\partial \phi(\mathbf{x}, t)}{\partial t}+F(\mathbf{x}, t)\|\nabla \phi(\mathbf{x}, t)\|=0
$$

where $F(\mathbf{x}, t)$ denotes the speed function of the level set. The original formulation for the speed function uses image gradient $[4,9]$. However, such speed function does not work well when the image is noisy as well as the cases in which the boundary of the desired object is not clear. Unfortunately, such characteristics are common in many of MRIs which makes it hard to benefit such speed function. To improve the performance of level set, the idea of integrating the region information instead of the image gradient into the level set speed function was proposed $[2,7]$. Motivated by this idea, TSL uses a threshold based speed function.

The level set speed function is $F=F_{0} \cdot F_{I}^{(i)}-\epsilon \kappa_{\phi}$, where $F_{0}$ is a constant propagation determined by a positive number and $F_{I}^{(i)}$ is defined based on image characteristics in the $i^{t h}$ iteration and causes the level set to finally attract to the tumor boundary. The smoothness parameter, $\kappa_{\phi}$, is the mean curvature of $\phi(\mathbf{x}, t)$ which prevents the level set leaking into many small noisy structures in the tumor region. The strength of this smoothing is controlled with a positive constant factor $\epsilon$. 
Intuitively, it is desired to have higher growing speed in a region where the tumor likelihood is higher and vice versa. TSL uses an speed function which takes this feature into account. In other words, TSL determines $F_{I}$ for each sample proportional to it's difference from the threshold so that the greater the difference the higher the speed. Let $T_{i}$ be the threshold associated with the $i^{t h}$ iteration. $F_{I}^{(i)}$ is defined for each sample based on its normalized difference form $T_{i}$ as follows,

$$
F_{I}^{(i)}(x, y, z)=\frac{\Delta}{2}\left[\frac{1+\operatorname{sgn}(\Delta)}{\max (\Delta)}-\frac{1-\operatorname{sgn}(\Delta)}{\min (\Delta)}\right]
$$

where $\Delta=I(x, y, z)-T_{i}$ and $\operatorname{sgn}$ is the signum function defined as follow,

$$
\operatorname{sgn}(x)=\left\{\begin{array}{cc}
1 & x>0 \\
0 & x=0 \\
-1 & x<0
\end{array}\right.
$$

It should be noted that the sign of $F_{I}^{(i)}$ indicates whether the sample is inside (+) or outside (-) the tumor region. In the Eq. (7), the first and second terms give the normalized level set speed for tumor and non tumor voxels respectively.

\subsection{Level Set initialization}

In TSL algorithm the initialization is performed inside the tumor region because the first estimation of the threshold, $T_{1}$ is calculated based on this initial set. Let define reference slice as one which includes a relatively large intersection with tumor compared to the other tumor-contained slices. It should be noted that the choice of this reference slice does not affect the process of the algorithm. Depending on the complexity of the tumor shape the initialization can be performed in the automatic or semi-automatic forms.

According to mathematics literature, a body is convex if and only if for any pair of points inside the body, the line segment which joins them lies entirely inside the body, otherwise the body is called concave [11]. For the convex tumor shape, TSL uses an spherical surface as an initial zero level set with the center which is placed automatically in the center of ROI. However, initialization for concave tumor is slightly more complicated so that multiple spheres may be required to achieve higher precision in the final results. These multiple spheres should be chosen independently and fairly spread on the entire tumor region and their centers are inserted manually. In both cases the initialization is performed so that the cross-section of the spheres with the reference slice is ensured to be within the tumor region. However it is not necessary for the other slices to satisfy this condition. $T_{1}$ is calculated by Eq. (3) just based on the selected tumor samples in the reference slice. Fig. 1 shows an example of such initializations.
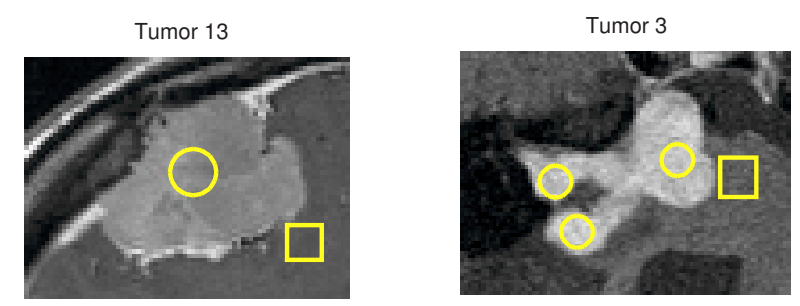

Figure 1. TSL initializations on the reference slices for the convex tumor (left), and concave tumor (right). Circles are the level set initialization and squares show the sampling from non-tumor region.

\subsection{Stopping criterion}

As described, TSL updates the threshold at each iteration based on the accepted tumor samples upto that iteration. At the tumor boundary, the negative level set speed for nontumor samples causes the TSL to reject them. Therefore rate of accepting new samples declines so that the variations of the threshold becomes negligible per iteration. Fig. 3 shows the variation of threshold versus iterations for two real MRIs. This fact, motivates to define the stopping criterion as follows.

Let define the stopping window of length $W_{s}$ so that it contains the thresholds associated with the last $W_{s}$ iterations. TSL stops when for $t_{s}$ consecutive iterations the variance of the thresholds in the stopping window becomes less than a very small number say $\varepsilon$. In fact, for the large enough values of $W_{s}$ and $t_{s}$ and a small value of $\varepsilon$ the overall performance of the TSL algorithm does not change significantly.

\section{TSL analysis}

In this part, firstly, a detailed discussion about threshold updating and its main parameter, $k$, is provided and then the modified TSL for segmentation of non-homogeneous tumors is presented.

\subsection{Threshold updating parameter}

The rate of convergence and the accuracy of segmentation are directly related to the choice of the threshold which is also determined by $k$. Therefore, the parameter $k$ plays a critical role in the overall performance of TSL algorithm. In the case where $k$ is chosen very small the level set may never grow while for relatively large values of $k$, convergence of TSL may not be possible.

In this section, two different schemes for appropriate choosing of $k$ are proposed. In the first scheme, the value of 
$k$ is kept fixed during the level set evolution procedure while in the second scheme, it is chosen adaptively as the level set grows. The adaptive scheme, although needs a slightly more complicated initialization helps to reduce the overall complexity of the algorithm.

According to Eq. (2), $k=1.5$ can provide a reasonable confidence level for the accepted samples. Hence, this value is used as the lower bound of $k$ in the following analysis. Now, take $k$ a relatively large value so that the threshold becomes very small. It is obvious that in such a case, TSL never converges because the samples of non-tumor region will be included in the level set evolution process. Therefore, there must exist a critical value of $k_{c}$ greater than 1.5 , thereafter TSL diverges. Since TSL achieves its best performance at $k_{c}$, the main goal of search-based scheme is to find an approximation of it. For this reason, the values of $k$ such that $1.5 \leq k$ is swept with a reasonably small step $s_{k}$.

The search-based scheme is simple but an exhaustive search must be performed in order to achieve an acceptable precision of $k_{c}$. The adaptive scheme tries to rectify this complexity just by an additional sampling from the nontumor region in the initialization phase. Such sampling although needs more user involvement, greatly reduces the complexity of computation. The shape of selected area in the non-tumor region is not important however it is required not to contain any voxels from tumor. Preferably, it should be selected near to the tumor boundary where the tumor and non-tumor intensities are more correlated as shown in Fig. 1. Assuming the same confidence level for both accepted tumor set and sampled non-tumor set in each iteration, a proper approximation of $k_{i}$ can be found providing non-overlapping confidence interval as follows,

$$
\begin{gathered}
\hat{\mu}_{N T}+k_{i} \hat{\sigma}_{N T}=\hat{\mu}_{i}-k_{i} \hat{\sigma}_{i} \\
\Rightarrow \quad k_{i}=\frac{\hat{\mu}_{i}-\hat{\mu}_{N T}}{\hat{\sigma}_{i}+\hat{\sigma}_{N T}}
\end{gathered}
$$

where $\left(\hat{\mu}_{N T}, \hat{\sigma}_{N T}\right)$ are the mean and standard deviation associated with the sampled non-tumor set.

Based on Eq.(9) the values of $k_{i}$ is proportional to the intensity level difference between the tumor and background. The higher the level of intensity difference, the greater the value of $k_{i}$ which means the higher confidence level around the tumor samples.

\subsection{Modified TSL for non-homogeneous tu- mors}

The described TSL is appropriate for the homogeneous tumors because in the threshold updating procedure, all accepted tumor samples are used. However, with the minor modifications in the threshold updating algorithm, TSL is able to segment the non-homogeneous tumors. In order to modify TSL algorithm, $\left(\hat{\mu}_{i}, \hat{\sigma}_{i}\right)$ can be calculated using only the accepted samples which are greater than the last threshold, $T_{i}$. Moreover, the level set must be initialized so that no sample from the non-homogeneity region in the reference slice is included in the initialized set. This modifications along with the effect of smoothness parameter in level set speed function enables TSL to segment the non-homogeneous tumors. It should be noted that the nonhomogeneity must be inside the tumor region and not on the boundary. Fig. 5 shows an example of such tumors which is successfully segmented by modified TSL.

\section{Simulation results and discussion}

In this section, firstly, the performance of the TSL algorithm for search-based scheme in comparison with the adaptive scheme is investigated. Then, the segmentation results of this algorithm on various MRIs are validated compared to the ground truthes. For this purpose, 13 multichannel 3D MRIs (T1W or CET1W) with the slice thickness of 2-3 mm are used. Among these MRIs, 5 tumors, labeled from 1 to 5 , are concave and the others, labeled from 6 to 13, are chosen convex. For the case of $3 \mathrm{~mm}$ slice thickness, the linear interpolation is done to get higher inter-slice resolution (1.5 $\mathrm{mm}$ ) and decrease the uncertainty between slices.

\subsection{Search-based vs. adaptive TSL}

In this section, the performance of the search-based TSL in comparison with its adaptive case is considered for both concave, Tumor 3 , and convex, Tumor 13 , tumors which are shown in Fig. 1. In the search-based scheme, parameter $k$ sweeps the values greater than 1.5 with the step size $s_{k}=$ 0.1 in order to approximate $k_{c}$.

In the search-based TSL, $k_{c}$ is found 1.7 and 2 for the convex and concave cases, respectively. As discussed, the greater value of $k_{c}$ for the concave case is expected because of the higher intensity level difference between tumor and background in this case.

For the adaptive TSL, the variations of $k$ versus the iteration number is shown for the same tumors in Fig. 2. In this case, the values of parameter $k$ are also also higher for the concave tumor which is consistent with the foregoing discussion. Another observation based on this figure is that the value of $k$ for both cases are monotonically decreasing up to almost constant values for each case which can be concluded from our discussion in Sec. 3.1. Interestingly, the final values of $k_{i}$ in both cases are close to their corresponding $k_{c}$ concluded from the search-based TSL.

Finally, comparing the threshold variation curves associated with both search-based and adaptive schemes as shown in Fig. 3, it is concluded that the convergence rate of the adaptive scheme is higher than that of the search-based 


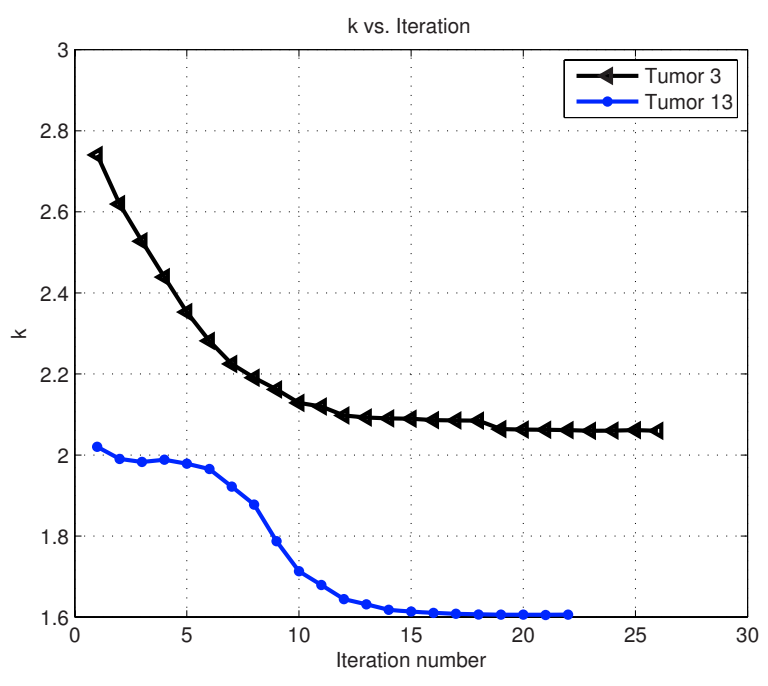

Figure 2. Variations of $k$ vs. iteration number using the adaptive TSL

scheme. This event can be justified through the larger number of samples which is selected from both tumor and nontumor regions in the initialization phase of adaptive scheme.

\subsection{Segmentation validation}

In this part, the results of 3D segmentation by TSL over 13 MRIs for both search-based and adaptive schemes are compared with the ground truths drawn by an expert. Fig. 4 shows the cross-sections of the extracted surfaces from tumors 3 and 13 with their corresponding MRI slices. This figure also shows the final extracted 3D tumor surfaces. It should be noted that these surfaces are the results of the adaptive scheme.

Since the manual segmentations were carried out in 2D, in order to facilitate comparison, the cross-sections of the resulted 3D surface (by TSL) with the MRI slices were considered for validation. Let $G T$ and $\Omega$ denote the set of tumor voxels in the ground truth and the set resulted from TSL, respectively. In this paper, two measures are used for comparison which are based on the volume overlap. One of them compares the volume of the intersection with the ground truth volume, $P M=\frac{\#(G T \cap \Omega)}{\# G T}$, and the other one compares it with the union volume [10], $J M=\frac{\#(G T \cap \Omega)}{\#(G T \cup \Omega)}$. The higher Percentage Match, $P M$, indicates the more true voxels contained by the results and the higher Jaccard Measure, $J M$, indicates that the results contain more true voxels while less false positive ones ${ }^{2}$. The validation of segmentation results are shown in Table 1.

\footnotetext{
${ }^{2}$ Voxels isolated as tumors by TSL but not in conformance with GT
}

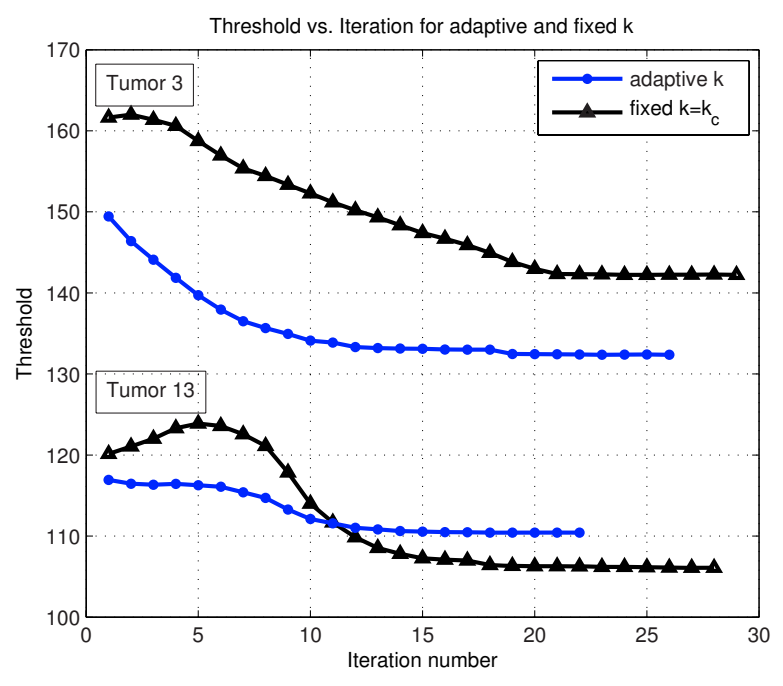

Figure 3. Threshold variation curves for the search-based and adaptive TSL

As mentioned, the first five tumors have concave body while the other tumors are convex. Table 1 shows the error of segmentation for concave tumors are almost higher than convex ones which can be justified through the complexity of the tumor shapes and the effect of smoothness parameter in the level set speed function. It can be seen that in the case of concave tumors, adaptive TSL gives better results compared to the search-based TSL. Whereas, for the convex tumors it is possible that search-based TSL performs better because of doing exhaustive search. Moreover, in the cases of tumors 8,11, 12 and 13, both search-based and adaptive TSL perform almost the same. Considering their respective MRIs, the reason is because the overlap between the tumor and non-tumor regions in these cases are relatively small.

\section{Conclusion}

This paper presents a new threshold-based approach, called TSL, for 3D tumor segmentation in brain MRIs. TSL uses level set as a deformable model and defines its speed function on the basis of intensity thresholding so that no explicit knowledge about the density functions of the tumor and non-tumor regions are required. Threshold is updated iteratively throughout the level set growing process using two schemes which are presented and their performance are compared to each other. The visualization and quantitative evaluations of the segmentation results demonstrate the effectiveness of our proposed approach. TSL performance is better for the cases where the intensity level difference between the tumor and non-tumor regions is higher. It can also segment non-homogenous tumors providing the non- 
Table 1. Quantitative validation of segmentation

\begin{tabular}{c|c|c|c|c|c|c|c|c|c|c|c|c|c|c}
\hline \hline & Tumor index & 1 & 2 & 3 & 4 & 5 & 6 & 7 & 8 & 9 & 10 & 11 & 12 & 13 \\
\hline$P M$ & search-based & 73 & 78 & 81 & 85 & 89 & 88.9 & 91 & 91.5 & 93.7 & 95 & 93 & 95 & 96.5 \\
$(\%)$ & adaptive & 80 & 87 & 87 & 91.6 & 93 & 93.5 & 96.2 & 90 & 91.6 & 92.4 & 95.3 & 95.7 & 95.7 \\
\hline \hline$J M$ & search-based & 72.8 & 75 & 79.8 & 80.7 & 87 & 88.5 & 90.6 & 89.6 & 91.8 & 86.4 & 92.9 & 90.6 & 88.5 \\
$(\%)$ & adaptive & 78 & 84 & 84.5 & 88.5 & 89 & 91 & 87.4 & 88 & 90.6 & 90.6 & 93 & 91 & 90.1 \\
\hline \hline
\end{tabular}
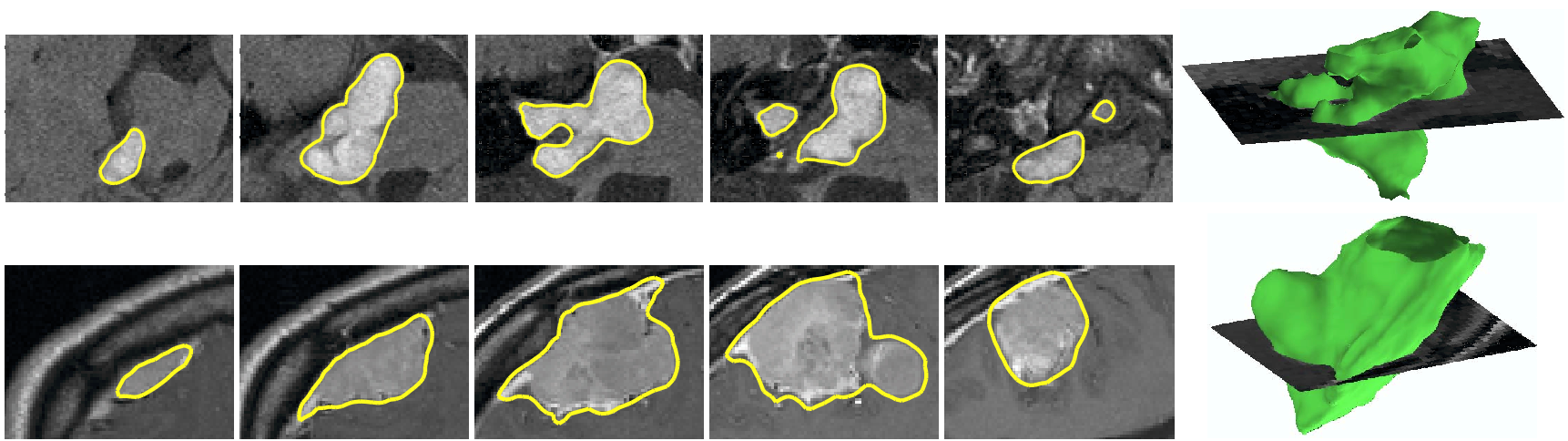

Figure 4. The cross-sections of the extracted surfaces with the MRI slices in addition to a 3D view of their corresponding surfaces crossed by their reference slices (the middle shown slices).

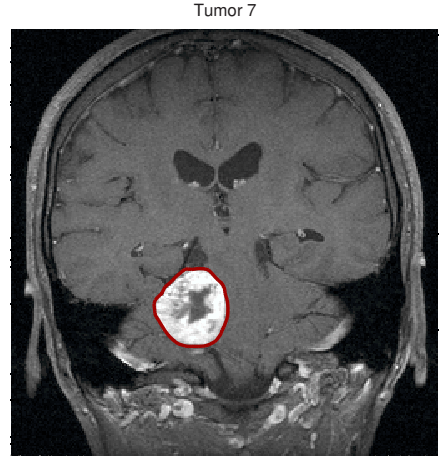

Figure 5. result of modified TSL on nonhomogeneous tumor.

homogeneity is within the tumor region. Moreover, TSL segments convex tumors with higher precision compared to concave ones. A localized approach to determine the threshold is part of our future work.

\section{References}

[1] V. Caselles, R. Kimmel, and G. Sapiro. Geodesic active contours. Int'l Journal of Computer Vision, 22(1):61-79, 1997.
[2] S. Ho, E. Bullitt, and G. Gerig. Levelset evolution with region competition: Automatic $3 \mathrm{~d}$ segmentation of brain tumors. In Proc. of Int'l Conf. on Pattern Recognition, pages 532-535, August 2002.

[3] A. Kichenassamy, A. Kumar, P. Olver, A. Tannenbaum, and A. Yezzi. Gradient flows and geometric active contour models. IEEE Int'l Conf. on Computer Vision, 1995.

[4] R. Malladi, J. A. Sethian, and B. C. Vemuri. Shape modeling with front propagation: A level set approach. IEEE Trans. on Pattern Analysis and Machine Intelligence, 17(2):158$175,1995$.

[5] Osher, Stanley, Fedkiw, and Ronald. Level set methods and dynamic implicit surfaces. Springer, 2003.

[6] B. Ostle and L. Malone. Statistics in research: basic concepts and techniques for research workers. Iowa State University Press, fourth edition, 1988.

[7] M. Prastawa, E. Bullitt, S. Ho, , and G. Gerig. A brain tumor segmentation framework based on outlier detection. Medical Image Analysis, 8(3):275-283, Sep 2004.

[8] S. Saini. Radiologic measurement of tumor size in clinical trials: past, present, and future. American Journal of Roentgenol, 176(2):333-334, February 2001.

[9] J. A. Sethian. Level Set Methods and Fast Marching Methods : Evolving Interfaces in Geometry, Fluid Mechanics, Computer Vision, and Materials Science.

[10] K. Teknomo. Similarity measurement. [Online]. Available: http://people.revoledu.com/kardi/tutorial/Similarity.

[11] E. W. Weisstein. Convex (concave). From MathWorldA Wolfram Web Resource, [Online]. Available: http://mathworld.wolfram.com/Convex.html. 